\title{
Filamentary structure formation in the Interstellar Radiation Field (ISRF)
}

\author{
Jingqi Miao, M. Lieu, Philip Cox, Tim Kinnear and Paul Cornwall \\ Centre for Astrophysics and Planetary Science (CAPS) \\ University of Kent, CT2 7NH, United Kingdom \\ email: j.miao@kent.ac.uk
}

\begin{abstract}
A new mechanism is proposed for the formation of filament/core structure by ISRF and clumpy molecular cloud interaction. The derived characterizes of the filament/core network is consistent with that produced by the compressive forcing turbulence model.
\end{abstract}

Keywords. ISM: clouds - ISM: evolution - ISM: structure

The filamentary networks revealed by Herschel over the entire range of galactic latitudes (e.g. Molinari et al. 2010) indicate a link to the surrounding ISRFs (e.g. Men'shchikov et al. 2010). We probe a new mechanism for ISRF(FUV) induced filamentary network formation by using an existing numerical code (Nelson \& Langer 1997).

The four columns in the middle panel in Fig. 1 (from left to right) are the cross section density profiles from four different simulations for a clumpy cloud of an initial 40 solar masses and radius of $1.2 \mathrm{pc}$, subject to FUV radiation fluxes of $0.2,0.6,1$ and 2 times the Habing constant respectively. A density contrast of 3 orders of magnitude is reached after 1.14, 1.07, 0.97 and 0.75 Myrs respectively. The right panel in Fig. 1 is the velocity vector field overlaid the cross section density profile corresponding to the second left column in the middle panel of the Fig. 1, which bears a resemblance to that produced by the compressive turbulent forcing model (Federrath et al. 2010).

Our simulation result shows that the interaction between ISRF and a clumpy molecular cloud can be taken as a possible mechanism for filamentary network formation. More simulation results, discussions and references can be found in a paper to be submitted.
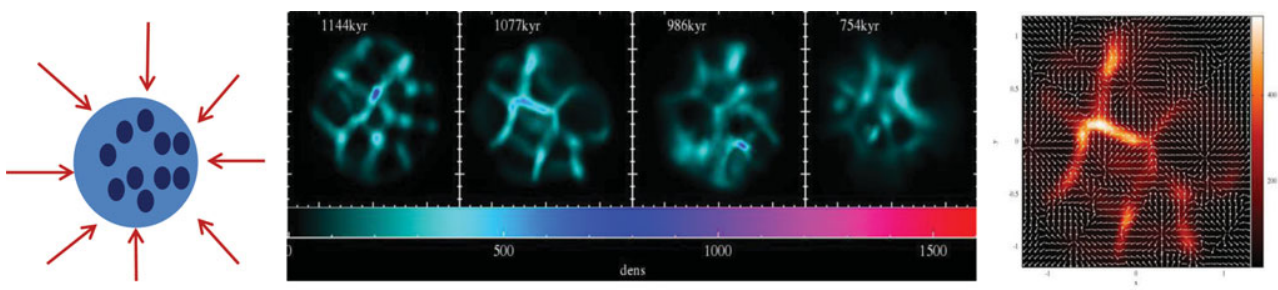

Figure 1. The left panel is the initial configuration of the simulation, the rest are the results.

\section{References}

Molinari, S. et al. 2010, A\&\&A, 518, L58

Men'shchikov, A. et al. 1999, A\&BA, 518, L103

Federrath, C., Roman-Duval, J., Klessen, R. S., Schmidt, W., \& Mac Low, M.-M. 2010, Aש̋A, 512,81

Nelson, Richard P. \& Langer, William D. 1997, ApJ, 482, 796 